\title{
The use of a lateral wedge insole to reduce knee loading when ascending and descending stairs in medial knee osteoarthritis patients
}

\author{
Amneh Z. Alshawabka ${ }^{\text {a }}$, Anmin Liu ${ }^{\text {b }}$, Sarah F. Tyson ${ }^{c}$, Richard K. Jones ${ }^{\text {b,* }}$ \\ a Faculty of Rehabilitation Sciences, University of Jordan, Jordan \\ b School of Health Sciences, University of Salford, UK \\ ' Stroke E' Vascular Research Centre, University of Manchester, UK
}

\section{A R T I C L E I N F O}

Article history:

Received 3 December 2013

Accepted 17 April 2014

\section{Keywords:}

Knee osteoarthritis

Adduction moment

Stair climbing

Lateral wedge insole

\begin{abstract}
A B S T R A C T
Background: Stair climbing is a challenging task to the elderly being the task with the first complaint in patients with mild to moderate knee osteoarthritis. Stair climbing results in around six times more compressive load transmitted through the knee joint than walking on level ground. The purpose of this study was to assess whether lateral wedge insoles would reduce medial compartment knee loading when ascending and descending stairs in patients with medial knee osteoarthritis.

Methods: Eight patients with medial knee osteoarthritis were tested in random order with and without a pair of $5^{\circ}$ off-the-shelf lateral wedge insoles for two separate activities (stair ascent and stair descent). Kinematic and kinetic data were collected for the lower extremity using a sixteen camera motion capture system and two force plates. Primary outcome measures were the external knee adduction moment and the knee adduction angular impulse.

Findings: During stair ascent and descent, lateral wedge insoles significantly $(P<0.05)$ reduced the 1 st peak external knee adduction moment in early stance (ascent 6.8\%, descent 8.4\%), the trough in mid stance (ascent $13 \%$, descent $10.7 \%$ ), 2nd peak in the late stance (ascent 15\%, descent $8.3 \%$ ) and the knee adduction angular impulse compared to the control (standard shoe) with large effect sizes (0.75-0.95).

Interpretation: In this first study on stairs, lateral wedge insoles consistently reduced the overall magnitude of medial compartment loading during stair ascent and descent. Further research is needed to determine the relationship of this with clinical results when ascending and descending stairs with lateral wedge insoles.
\end{abstract}

(C) 2014 Elsevier Ltd. All rights reserved.

\section{Introduction}

Stair climbing is a common and frequent dynamic activity in daily living which is more biomechanically and physiologically challenging than typical walking tasks and is considered as one of the most difficult tasks for the elderly (Hemenway et al., 1994; Tinetti et al., 1994). Moreover, stair climbing was reported as the first complaint task in patients with mild to moderate knee osteoarthritis (OA) with the highest pain score (Costigan et al., 2002). Stair climbing demands, compared to walking on level ground, a greater range of motion in the lower extremity accompanied by about six times more compressive load on the knee joint (Andriacchi et al., 1980). During walking gait, greater loading is seen in the medial compartment of the knee with the asymmetric load distribution due to the external knee adduction moment (EKAM). There is increasing evidence that the EKAM is a

\footnotetext{
* Corresponding author at: Room PO42 Brian Blatchford Building, School of Health Sciences, University of Salford, UK.

E-mail address: r.k.jones@salford.ac.uk (R.K. Jones).
}

reliable predictor of osteoarthritis progression and severity (Bennell et al., 2011; Miyazaki et al., 2002) and can be used as an indirect measure of medial joint loading (Zhao et al., 2007). During walking, individuals with knee OA have shown 35\% higher peak EKAM as compared with healthy subjects (Thorp et al., 2006). Additionally, in patients with knee OA, the first peak of EKAM was $19 \%, 18 \%$, and $36 \%$ higher than the second peak of EKAM in walking, ascending, descending stairs, respectively (Guo et al., 2007), with the largest peak of EKAM recorded during descending stairs followed by ascending stairs and then level walking (Guo et al., 2007). In a longitudinal study, 39\% higher EKAMs were present during stair descent in individuals who developed knee OA 3-5 years later (Amin et al., 2004). Therefore, reducing the EKAM has become the objective of early and conservative treatment in an attempt not only to reduce pain and maintain function but also to attempt in arresting disease progression.

A lateral wedge insole is a cheap and simple intervention (Kerrigan et al., 2002), and it has been found that it offers reduced medial compartment loading between $5.3 \%$ and $9 \%$ in the affected and contralateral knee in patients with medial knee OA (Hinman et al., 2008; Jones et al., 2013a; Kakihana et al., 2007). Lateral wedges are theorised to modify 
load by changing the kinematics and kinetics of the ankle/subtalar joint. Previously, investigators have reported that a lateral wedge insole increases foot pronation which aligns the tibia and femur into a more upright position (Crenshaw et al., 2000; Jones et al., 2013b; Kerrigan et al., 2002; Maly et al., 2002). This along with an increased lateral centre of pressure and valgus ankle moments at the ankle resulted in less medial knee loading (Kakihana et al., 2005; Sasaki and Yasuda, 1987; Yasuda and Sasaki, 1987).

Whilst there is a plethora of studies on walking, there are limited studies investigating the effect of lateral wedge insoles on stair climbing with only one previous study investigating the immediate (after 1 week) and short term effects (after 12 weeks) of customised lateral wedge insoles with a neutral insert during stair descent. No reductions in EKAM were seen when using the lateral wedge insole but they did report an increase in EKAM at week 1 and week 12 with the lateral wedge insoles (Wallace et al., 2007).

To the author's knowledge, the effects of using a lateral wedge insole on the external knee adduction moment for patients with medial knee OA during ascending stairs have not been evaluated, nor fully evaluated for descending stairs. In the current study, an off-the-shelf lateral wedge insole (prefabricated according to fixed moulds independent of the variability amongst patients) was used to cancel out the variability of person-specific lateral wedge insoles which results from the inability of the manufacturing orthotist to accurately reproduce insoles with the same characteristics. Therefore, the purpose of this study was to determine whether an off-the-shelf lateral wedge insole reduced knee loading in the medial compartment during ascending and descending stairs in patients with medial knee OA.

\section{Methods}

This study was a randomised crossover design study comparing two conditions whilst ascending and descending stairs.

The Research Ethics Panel at the University of Salford approved the study. The inclusion criteria for participation were: over 40 years of age, medial knee osteoarthritis grades 2-3 (Kellgren and Lawrence, 1957) with greater medial narrowing than lateral, ability to walk and ascend and descend stairs without aids and/or assistance, no musculoskeletal complaints in the lower limb and has not worn assistive devices for medial knee OA. Patients with symptomatic evidence of lateral compartment or patellofemoral $\mathrm{OA}$, rheumatoid arthritis, surgery within the past six months, previous stroke, hip or ankle symptoms, or a body mass index of above 35 were excluded. A sample of eight patients with medial knee OA ( 5 females, 3 males), age (47.38 (SD 3.02) years), height (1.69 (0.85) metre), body mass (66.13 (11.49) kg), Kellgren-Lawrence Scale ( 6 patients grade II, 2 patients grade III) participated in the study and all the patients had greater medial than lateral joint space narrowing.

To minimise the influence of footwear, all subjects wore the same type of standard shoe (ECCO Zen) which provided a baseline dataset for the footwear conditions. Two conditions were assessed, a control condition (with a neutral flat insert) and a lateral wedge insole. The lateral wedge insoles (Fig. 1) are off-the-shelf insoles made from a comfortable and flexible material (SureStep-Control ${ }^{\mathrm{TM}}$, with a medium density Shore A 70, SalfordInsole, Nuneaton, UK). The insoles (SalfordInsole, Nuneaton, UK) were full length lateral wedge insoles constructed with a medial arch, with a $5^{\circ}$ lateral wedge used in previous studies (Forghany et al., 2010; Jones et al., 2013a,b).

Kinematic data were collected for the lower extremity using a 16 camera Qualisys OQUS motion capture system (Qualisys, Gothenburg, Sweden) sampling at $100 \mathrm{~Hz}$. Passive retro-reflective markers were placed at the following anatomical sites: anterior superior iliac spine, posterior superior iliac spine, greater trochanter, medial and lateral femoral epicondyle, head of fibula, tibial tuberosity, and medial and lateral malleloli. Markers were glued to the heel and forefoot of the shoe. The CAST protocol (Cappozzo et al., 1995) was used to allow for segmental kinematics to be tracked in six-degrees of freedom and all cluster markers on the shank, thigh and pelvis stayed in place throughout each data collection session.

Kinetic data $(200 \mathrm{~Hz})$ were collected using two AMTI (BP400600) (AMTI, Boston, USA) force plates. The custom stairs (AMTI stairs (Della Croce and Bonato, 2007)) were securely fixed to the two force plates with bolts. This allows independent measurement of forces on each stair separately without the need to modify the existing setup in the laboratory. For example, when a contact is made on the first stair, data is recorded from the second force platform; when a contact is made on the second stair, data is recorded from the first force platform; and when a contact is made with the third stair, data is recorded from the second force platform (Fig. 2). This allowed gait events to be determined from the force platform data. A force structure was built within Visual 3D and CalTester (Holden et al., 2003) was performed ensuring that the force plates' location and laboratory frame were coincident to ensure accurate calculation of joint kinetics.

Prior to data collection, all subjects had the opportunity to become familiar with the interventions, and they were asked to walk and climb stairs for 10 min to ensure proper fit (of the shoes and lateral wedge insole) and comfort level. Each participant performed five trials

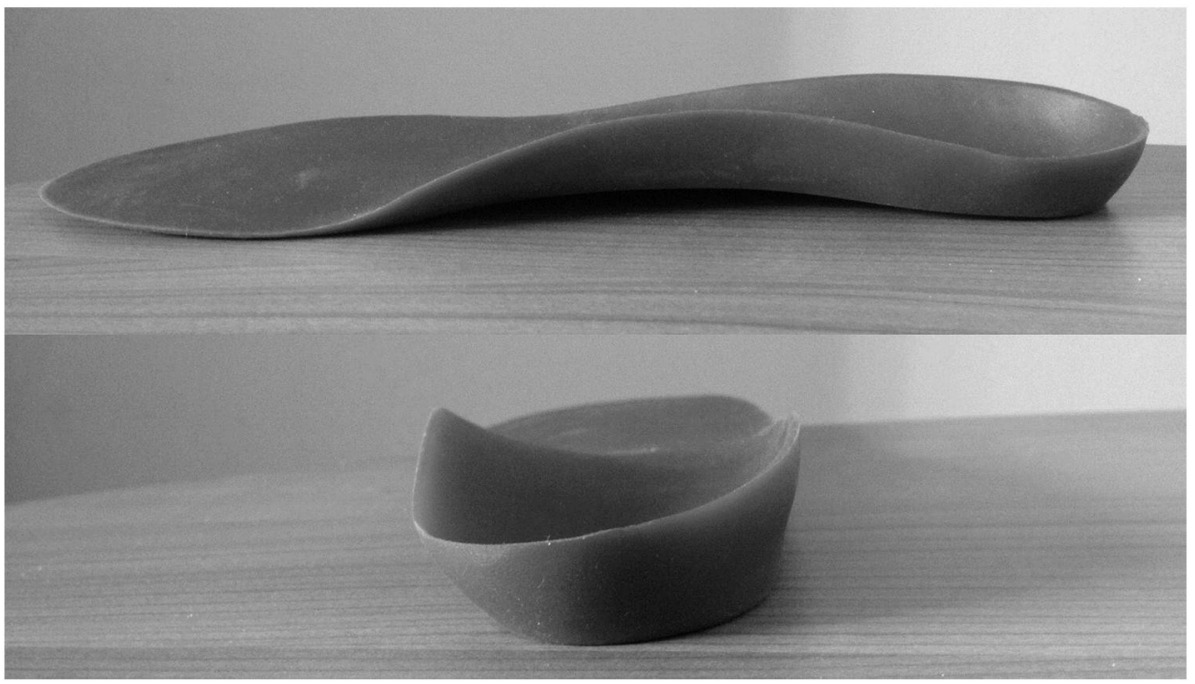

Fig. 1. Lateral wedge insole. 


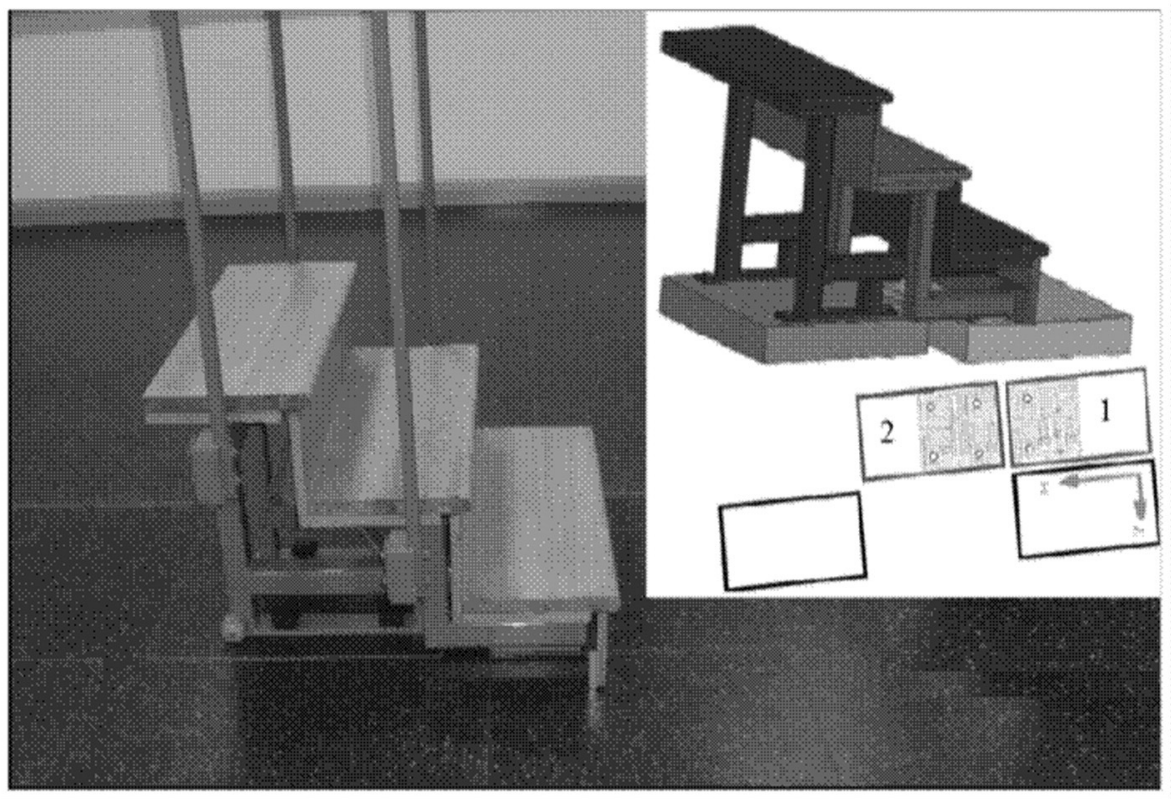

Fig. 2. Stair configuration for tests.

of stairs ascent and descent at a self-selected speed starting every trial with the same foot and without using the handrails. For each ascending trial, subjects stood just in front of the first force platform and took an initial step on the floor, the subjects then ascended the three stairs in a step-over-step manner (Reid et al., 2007). When the subjects reached the third stair, they were asked to stand upright, turn around and then
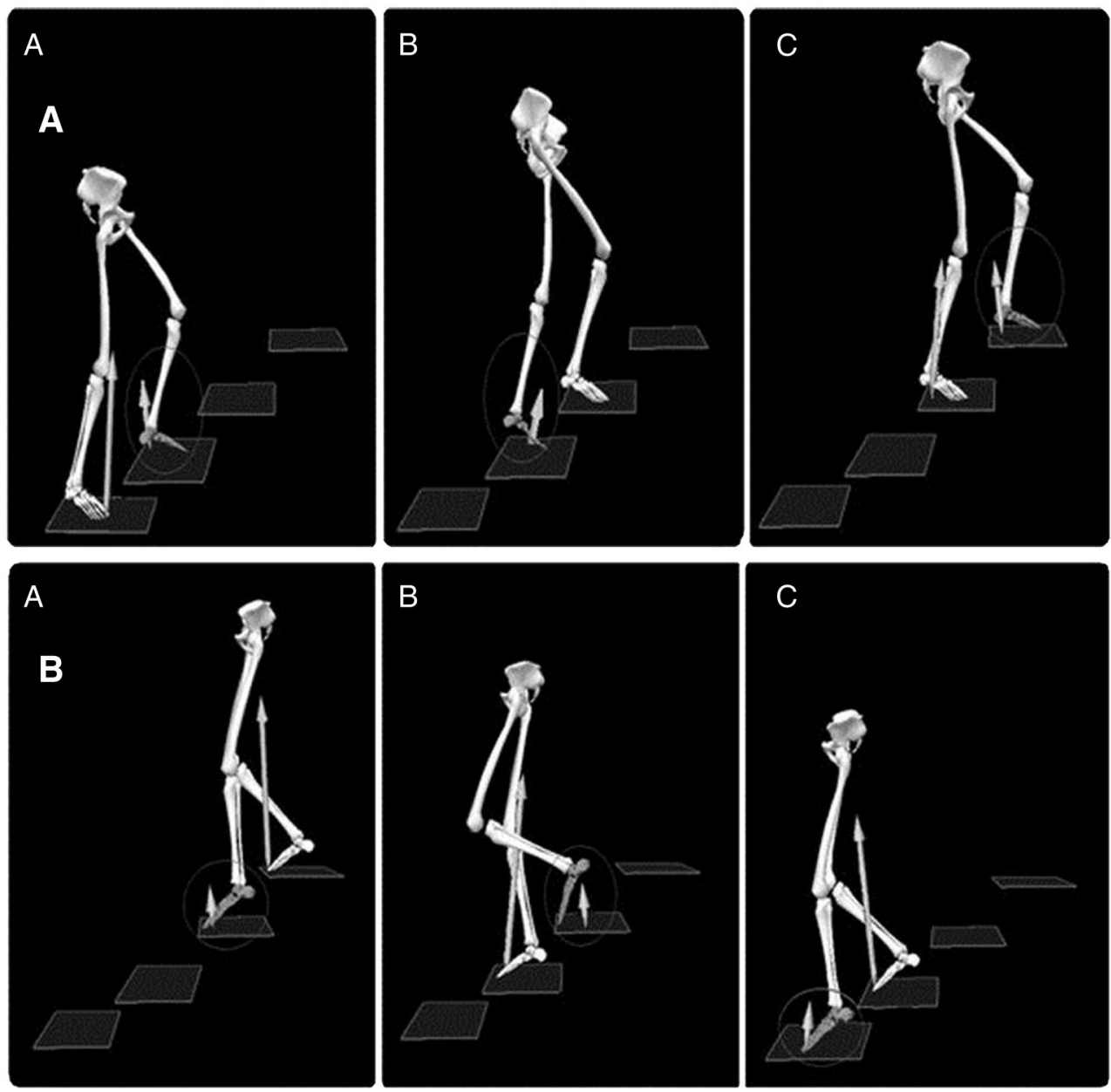

Fig. 3. Stair climbing definitions for data analysis.

Please cite this article as: Alshawabka, A.Z., et al., The use of a lateral wedge insole to reduce knee loading when ascending and descending stairs in medial knee osteoarthritis patients, Clin. Biomech. (2014), http://dx.doi.org/10.1016/j.clinbiomech.2014.04.011 
to descend the three stairs in the step-over-step manner. The descending trial ended when the subjects reached the floor, with both feet off the force platform. This process was repeated for each condition and the participants rested after every test condition.

Post-processing calculation of the kinematic and kinetic data was conducted using Visual 3D software (C-Motion Inc., Rockville, MD, USA). All lower extremity segments were modelled as rigid bodies. Anatomical frames were defined by landmarks positioned at the medial and lateral borders of the joint; from each segment a right handed coordinate system was defined. Joint kinematics were calculated using an X-Y-Z Euler rotation sequence. Joint kinetic data were calculated using three-dimensional inverse dynamics, and the external joint moment data were normalised to body mass ( $\mathrm{Nm} / \mathrm{kg}$ ).

The ascending stair gait cycle was chosen to be ascending from stair 1 to stair 3 (Fig. 3); descending stair gait cycle was chosen to be descending from stair 2 to the floor (Fig. 3 ). The ascending stair gait cycle from floor to stair 2 and the descending stair gait cycle from stair 3 to stair 1 were excluded, as these stair gait cycles are considered transitional cycles from walking to ascending stairs and from standing still to descending stair, respectively. However, using these stair gait cycles allows comparison with the study by Wallace et al. (2007) who used the same stair gait cycles.
The biomechanical parameters of interest were the External Knee Adduction Moment observed at (A) early stance (first peak of EKAM), (B) mid stance (EKAM trough), (C) late stance (second peak of EKAM) (Jones et al., 2013b) and (D) The area under the adduction moment curve represents the Knee Adduction Angular Impulse (KAAI) (Thorp et al., 2006). In addition, ankle eversion and the centre of pressure of the foot (relative to the local coordinate system of the foot) during stance phase were also assessed to understand the influence of the lateral wedge insoles. All kinetic and kinematic parameters were based on the mean of the maximum/minimum peak values across the trials for each condition and for each participant. The Shapiro-Wilk test of normality showed that the majority of the variables tested in this study were normally distributed and therefore a paired t-test was performed comparing the means between the conditions at the 95\% confidence interval. Effect sizes were also estimated $(\sqrt{ }$ (F-statistic / F-statistic / degrees of freedom)) and interpreted based on the method of Cohen (1992) where small $\geq 0.10$, medium $\geq 0.30$ and large $\geq 0.50$.

\section{Results}

There were no significant differences in speed ( 0.45 vs. $0.44 ; 0.46$ vs. $0.45 \mathrm{~m} / \mathrm{s}$ ), percentage stance time ( 63.8 vs. $63.9 ; 63.3$ vs. $63.9 \%$ gait
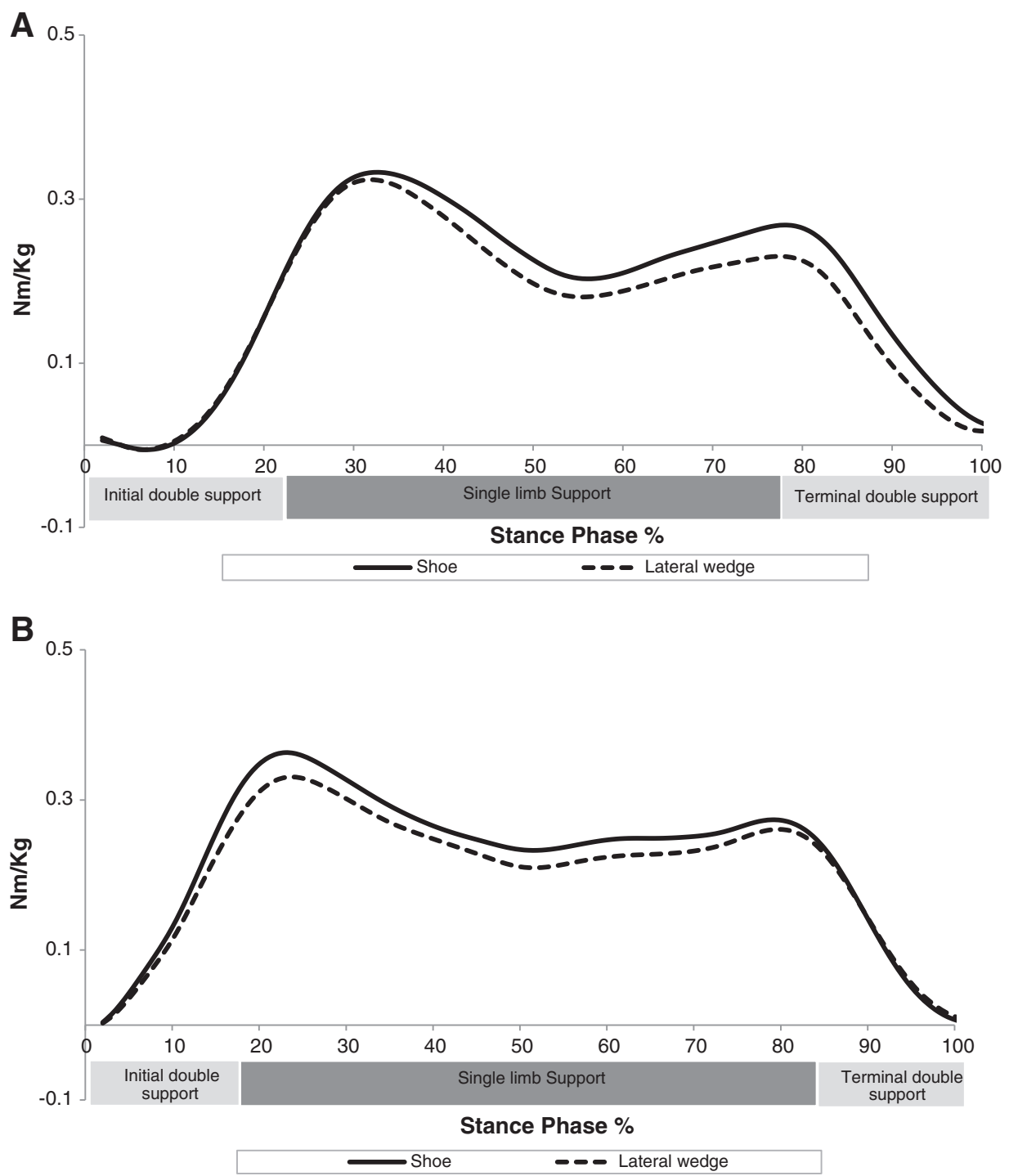

Fig. 4. Population mean EKAM during ascending (A) and descending (B) stairs with and without lateral wedge insoles. 
Table 1

EKAM, KAAI and knee flexor moment during ascending and descending stairs.

\begin{tabular}{|c|c|c|c|c|c|}
\hline \multirow[t]{2}{*}{ Parameters } & & \multicolumn{2}{|l|}{ Mean (SD) } & \multicolumn{2}{|c|}{ Control vs. lateral wedge } \\
\hline & & Control & Lateral wedge & $P$ value & Effect size \\
\hline \multirow[t]{2}{*}{ EKAM (early stance) (Nm/kg) } & Ascent & $0.39(0.03)$ & $0.37(0.02)$ & 0.005 & 0.88 \\
\hline & Descent & $0.37(0.06)$ & $0.33(0.05)$ & 0.001 & 0.93 \\
\hline \multirow{2}{*}{ EKAM (mid stance) (Nm/kg) } & Ascent & $0.19(0.05)$ & $0.16(0.06)$ & 0.014 & 0.84 \\
\hline & Descent & $0.23(0.07)$ & $0.21(0.07)$ & 0.002 & 0.91 \\
\hline \multirow[t]{2}{*}{ EKAM (late stance) (Nm/kg) } & Ascent & $0.28(0.07)$ & $0.24(0.06)$ & 0.043 & 0.75 \\
\hline & Descent & $0.28(0.05)$ & $0.26(0.06)$ & 0.001 & 0.94 \\
\hline \multirow[t]{2}{*}{$\mathrm{KAAI}(\mathrm{Nm} / \mathrm{kg} * \mathrm{~s})$} & Ascent & $0.19(0.06)$ & $0.17(0.06)$ & 0.003 & 0.90 \\
\hline & Descent & $0.20(0.04)$ & $0.19(0.04)$ & 0.006 & 0.88 \\
\hline \multirow{2}{*}{ Knee flexor moment $(\mathrm{Nm} / \mathrm{kg})$} & Ascent & $1.06(0.07)$ & $1.01(0.06)$ & 0.002 & 0.92 \\
\hline & Descent & $1.14(0.08)$ & $1.12(0.08)$ & 0.041 & 0.49 \\
\hline
\end{tabular}

cycle), nor in single limb support percentage time ( 0.36 vs. 0.36 ; 0.39 vs. $0.39 \%$ gait cycle) between the control and lateral wedge conditions during stair ascent and descent respectively.

In the early stance phase, the first peak of the EKAM was significantly reduced (Fig. 4) with the lateral wedge insole during both stair ascent and descent than the control condition (Table 1). The lateral wedge insole significantly reduced the first peak of the EKAM by on average $6.8 \%$ during stair ascent $(P<0.01)$ and $8.4 \%$ during stair descent $(P<0.001)$ when compared to the control condition (maximum reduction $12.4 \%$ in stair ascent and $12.5 \%$ in stair descent, minimum reduction $1.4 \%$ during stair ascent and 5\% during stair descent). The mean value of EKAM during mid stance (EKAM trough) was significantly reduced with the lateral wedge insole during both the ascent $(P<0.05)$ and descent $(P<0.01)$ compared to the control condition. When comparing the lateral wedge insole and control, the EKAM trough was reduced by 13\% during stair ascent and 10.7\% during stair descent (maximum reduction was $28 \%$ in stair ascent and $19.6 \%$ in stair descent, minimum reduction was $5 \%$ during stair ascent and $5 \%$ during stair descent). In late stance phase, the second peak of the EKAM was significantly reduced with the lateral wedge insole during stair ascent $(P<0.05)$ and descent $(P<0.01)$ than the control condition. When lateral wedge insole and control conditions were compared, the second peak of the EKAM was reduced by $15 \%$ during stair ascent and $8.34 \%$ during stair descent (maximum was $16 \%$ in stair ascent and $17 \%$ in stair descent, minimum reduction was $4 \%$ during stair ascent and 3.6\% during stair descent). The effect sizes during the three time points were all above 0.7 demonstrating high effects.

As expected, the KAAI was significantly reduced with lateral wedge insole during both stair ascent $(P<0.01)$ and stair descent $(P<0.01)$ than control condition (Table 1$)$. There were significant changes in the sagittal plane knee moments between the lateral wedge insole and the control condition during both stair ascent $(P<0.05)$ and stair descent $(P<0.05)$.

In regard to the factors which may have caused the change in EKAM, there was a significantly greater degree of peak ankle/subtalar eversion and a greater lateral centre of pressure trajectory (during early, mid and late stances) with a lateral wedge than in the control condition during both stair ascent and stair descent $(P<0.05)$ (Table 2$)$.

\section{Discussion}

This is the first study that has investigated the use of lateral wedge insoles on ascending and descending stairs in medial knee OA patients and has identified that medial compartment knee loading was significantly reduced during stair ascent and descent with a lateral wedge insole. The overall reductions seen in medial compartment knee loading (with the lateral wedge insoles) were greater for stair descent than stair ascent and reflected the increased loads being placed on the medial aspect of the joint during stair descent (Costigan et al., 2002; Whatling et al., 2009).

There has been one previous study of stair descent with lateral wedge insoles which has conflicting results (Wallace et al., 2007) and showed an increase at the first peak EKAM during stair descent. One reason for this discrepancy may be due to the custom insole used in the previous study and inter-subject differences but further analysis is limited due to the unavailability of a published article. This discrepancy is expected to result from the inability of the orthotist to reproduce identical insoles as they are customised to the patient's, although the authors did use a consistent $7^{\circ}$ inclination.

Studies have shown that lateral wedge insoles significantly reduce 1st peak external knee adduction moments during level walking but there is a conjecture in whether they reduce 2 nd peak external knee adduction moments with authors showing a decrease and an increase (Hurwitz et al., 2002; Mundermann et al., 2005). The current study has shown that the lateral wedge insole consistently reduced the external knee adduction moment during the 1st peak, trough and second peak of stance phase during stair ascent and descent. This demonstrates that medial compartment of the knee loading was reduced throughout the whole of the stance phase with the knee adduction angular impulse reduction confirming this. Previous literature has shown that there are

Table 2

Ankle eversion angle, centre of pressure and ankle valgus moment during ascending and descending stairs.

\begin{tabular}{|c|c|c|c|c|c|}
\hline \multirow[t]{2}{*}{ Parameters } & & \multicolumn{2}{|l|}{ Mean (SD) } & \multicolumn{2}{|c|}{ Control vs. lateral wedge } \\
\hline & & Control & Lateral wedge & $P$ value & Effect size \\
\hline \multirow[t]{2}{*}{ Peak ankle eversion (degrees) } & Ascent & $-4.41(0.21)$ & $-4.82(0.25)$ & 0.008 & 0.52 \\
\hline & Descent & $4.43(0.42)$ & $5.11(0.52)$ & 0.003 & 0.66 \\
\hline \multirow[t]{2}{*}{ Centre of pressure (early stance) (M) } & Ascent & $0.009(0.004)$ & $0.006(0.003)$ & 0.008 & 0.91 \\
\hline & Descent & $0.007(0.001)$ & $0.006(0.001)$ & 0.039 & 0.33 \\
\hline \multirow[t]{2}{*}{ Centre of pressure (mid stance) (M) } & Ascent & $0.009(0.003)$ & $0.006(0.001)$ & 0.013 & 0.77 \\
\hline & Descent & $0.007(0.001)$ & $0.005(0.001)$ & 0.006 & 0.81 \\
\hline \multirow[t]{2}{*}{ Centre of pressure (late stance) (M) } & Ascent & $0.010(0.003)$ & $0.008(0.002)$ & 0.048 & 0.59 \\
\hline & Descent & $0.013(0.001)$ & $0.012(0.001)$ & 0.005 & 0.83 \\
\hline \multirow[t]{2}{*}{ Ankle valgus moment $(\mathrm{Nm} / \mathrm{kg})$} & Ascent & $0.15(0.07)$ & $0.18(0.08)$ & 0.000 & 0.89 \\
\hline & Descent & $0.18(0.07)$ & $0.19(0.07)$ & 0.000 & 0.92 \\
\hline
\end{tabular}


some individuals who increase their EKAM (Kakihana et al., Hinman et al.) when on lateral wedge treatment but in this study all participants reduced their EKAM during stair ascent and descent and this is probably due to the more stable pattern of the task in stair climbing. Walter et al. (2010) reflected that a reduction in EKAM does not necessarily reduce medial contact force if a corresponding increase in knee flexion moment was seen but in this study a reduction on knee flexion moment was seen for this variable and therefore it would be assumed, based on previous literature (Zhao et al., 2007) that a reduction in medial contact force would have occurred.

From previous literature, the reduction in the EKAM when using lateral wedge insoles has been suggested to be the result of three main factors; increased eversion of the ankle/subtalar joint complex (assumed ankle joint) (Forghany et al., 2010), increased ankle valgus moment and the laterally located trajectory of the COP (Kakihana et al., 2004). However, no such mechanism has been detailed for stair ambulation. During walking on level ground, ankle eversion has been shown to be increased when wearing lateral wedge insoles (Forghany et al., 2010; Kakihana et al., 2004; Kerrigan et al., 2002) by changing the orientation of the foot relative to the floor (Yasuda and Sasaki, 1987). In the current study the lateral wedge insole increased the peak eversion angle during both ascending and descending stairs with a corresponding increase in ankle valgus moment. Although the increased eversion in the ankle joint and increased valgus moment can help alleviate the medial loading in patients with medial knee OA, discomfort due to high forces on the medial side of the foot (Kakihana et al., 2005) can develop with long term use of a lateral wedge insole from the increased ankle valgus moment (Rubin and Menz, 2005). However, the inclusion of the medial arch support in the lateral wedge insole used in the current study may prevent this discomfort as the medial arch redistributes forces evenly on the lower surface of the foot (Hiraoka et al., 2002; Nakajima et al., 2009), and has been shown to be more comfortable than a standard lateral wedge (Jones et al., 2013b). Similar to level ground walking (Kakihana et al., 2004; Keating et al., 1993) a lateral movement of the centre of pressure trajectory was seen during stair ascent and descent, in which the ground reaction force is shifted closer to the centre of the knee thereby reducing the EKAM (Andrews et al., 1996; Andriacchi, 1994). Recent studies (Hinman et al., 2008; Hunt et al., 2008) have shown that the lever arm change is a major factor in reducing the EKAM but they have not taken into account the role of the foot and ankle. Therefore, further analysis into the exact mechanism of the reduction in EKAM is needed when ascending and descending stairs in a larger population.

As with any study, this study had its limitations and the major ones were the small sample size and immediate assessment of the interventions. Whilst a small sample size was used for the study, excellent effect sizes were seen with the lateral wedge insoles for the primary outcome measure of the external knee adduction moment. This study only examined the immediate effects of lateral wedge insoles on stair ascent and descent and as with any intervention requiring persistent use, a longer term evaluation is required to capture both the true benefit and possible adverse effects of using lateral wedge insoles in individuals with medial knee OA. Whilst the study by Wallace et al. (2007) demonstrated increased EKAMs they did report a reduction in pain levels when descending stairs. Pain levels were not recorded in this study and therefore, it could not be concluded clinically if the reduction in knee loading during ascending and descending stairs resulted in an alleviation of symptoms. A further study is currently underway in investigating this relationship. Additionally, we did not record any clinical range of motion at the ankle joint, and although we saw a reduction in the ankle coronal plane angle, future studies should consider this.

In conclusion, this study has shown that lateral wedge insoles reduce the EKAM immediately whilst ascending and descending stairs for patients with medial knee OA. As EKAM reduction has been strongly linked to the unloading of the medial knee compartment and thereby reduced progression of disease in patients with medial knee $\mathrm{OA}$, a lateral wedge insole may be an effective conservative treatment for not only walking but also for stair ascent and descent. This has implications for patient care as stair climbing is a frequent everyday activity and individuals with medial knee OA have greater difficulty performing these tasks, so these devices would be a recommended biomechanical intervention for these activities. However, future clinical trials are needed to understand the change in pain during these activities.

\section{References}

Amin, S., Luepongsak, N., Mcgibbon, C.A., Lavalley, M.P., Krebs, D.E., Felson, D.T., 2004 Knee adduction moment and development of chronic knee pain in elders. Arthritis Care Res. 51, 371-376.

Andrews, M., Noyes, F.R., Hewett, T.E., Andriacchi, T.P., 1996. Lower limb alignment and foot angle are related to stance phase knee adduction in normal subjects: a critical analysis of the reliability of gait analysis data. J. Orthop. Res. 14, 289-295.

Andriacchi, T.P., 1994. Dynamics of knee malalignment. Orthop. Clin. N. Am. 25, 395-403.

Andriacchi, T.P., Andersson, G.B., Fermier, R.W., Stern, D., Galante, J.O., 1980. A study of lower-limb mechanics during stair-climbing. J. Bone Joint Surg. Am. 62, 749.

Bennell, K.L., Bowles, K.A., Wang, Y., Cicuttini, F., Davies-Tuck, M., Hinman, R.S., 2011 Higher dynamic medial load predicts greater cartilage loss over 12 months in medial knee osteoarthritis. Ann. Rheum. Dis. 70 (10), 1770-1774.

Cappozzo, A., Catani, F., Croce, U.D., Leardini, A., 1995. Position and orientation in space of bones during movement: anatomical frame definition and determination. Clin. Biomech. 10 (4), 171-178.

Cohen, J., 1992. A power primer. Psychol. Bull. 112, 155.

Costigan, P.A., Deluzio, K.J., Wyss, U.P., 2002. Knee and hip kinetics during normal stair climbing. Gait Posture 16, 31-37.

Crenshaw, S.J., Pollo, F.E., Calton, E.F., 2000. Effects of lateral-wedged insoles on kinetics at the knee. Clin. Orthop. Relat. Res. 375, 185.

Della Croce, U., Bonato, P., 2007. A novel design for an instrumented stairway. J. Biomech. 40, 702-704.

Forghany, S., Jones, R., Preece, S., Nester, C., Tyson, S., 2010. Early observations of the effects of lateral wedge orthoses on lower limb muscle length and potential for exacerbating spasticity. Prosthetics Orthot. Int. 34, 319.

Guo, M., Axe, M.J., Manal, K., 2007. The influence of foot progression angle on the knee adduction moment during walking and stair climbing in pain free individuals with knee osteoarthritis. Gait Posture 26, 436-441.

Hemenway, D., Solnick, S.J., Koeck, C., Kytir, J., 1994. The incidence of stairway injuries in Austria. Accid. Anal. Prev. 26, 675-679.

Hinman, R.S., Payne, C., Metcalf, B.R., Wrigley, T.V., Bennell, K.L., 2008. Lateral wedges in knee osteoarthritis: what are their immediate clinical and biomechanical effects and can these predict a three month clinical outcome? Arthritis Rheum. 59 (3) $408-415$.

Hiraoka, H., Nakajima, K., Oda, H., 2002. Non-surgical treatments for gonarthrosis. Clin. Calcium 12, 92.

Holden, J.P., Selbie, W.S., Stanhope, S.J., 2003. A proposed test to support the clinical movement analysis laboratory accreditation process. Gait Posture 17, 205-213.

Hunt, M., Birmingham, T., Bryant, D., Jones, I., Giffin, J., Jenkyn, T., Vandervoort, A., 2008. Lateral trunk lean explains variation in dynamic knee joint load in patients with medial compartment knee osteoarthritis. Osteoarthritis Cartilage 16, 591-599.

Hurwitz, D.E., Ryals, A.B., Case, J.P., Block, J.A., Andriacchi, T.P., 2002. The knee adduction moment during gait in subjects with knee osteoarthritis is more closely correlated with static alignment than radiographic disease severity, toe out angle and pain. J. Orthop. Res. 20, 101-107.

Jones, R.K., Chapman, G.J., Findlow, A.H., Parkes, M.J., Forsythe, L., Felson, D.T., 2013a. A new approach to prevention of knee osteoarthritis: reducing medial load in the contralateral knee. J. Rheumatol. 40 (3), 309-315.

Jones, R.K., Zhang, M., Laxton, P., Findlow, A.H., Liu, A., 2013b. The biomechanical effects of a new design of lateral wedge insole on the knee and ankle during walking. Hum. Mov. Sci. 32 (4), 596-604.

Kakihana, W., Akai, M., Yamasaki, N., Takashima, T., Nakazawa, K., 2004. Changes of joint moments in the gait of normal subjects wearing laterally wedged insoles. Am. J. Phys. Med. Rehabil. 83, 273.

Kakihana, W., Akai, M., Nakazawa, K., Takashima, T., Naito, K., Torii, S., 2005. Effects of laterally wedged insoles on knee and subtalar joint moments. Arch. Phys. Med. Rehabil. 86, 1465-1471.

Kakihana, W., Akai, M., Nakazawa, K., Naito, K., Torii, S., 2007. Inconsistent knee varus moment reduction caused by a lateral wedge in knee osteoarthritis. Am. J. Phys. Med. Rehabil. 86 (6), 446-454

Keating, E.M., Faris, P.M., Ritter, M.A., Kane, J., 1993. Use of lateral heel and sole wedges in the treatment of medial osteoarthritis of the knee. Orthop. Rev. 22, 921.

Kellgren, J., Lawrence, J., 1957. Radiological assessment of osteo-arthrosis. Ann. Rheum. Dis. 16, 494-502.

Kerrigan, D.C., Lelas, J.L., Goggins, J., Merriman, G.J., Kaplan, R.J., Felson, D.T., 2002. Effectiveness of a lateral-wedge insole on knee varus torque in patients with knee osteoarthritis. Arch. Phys. Med. Rehabil. 83, 889-893.

Maly, M.R., Culham, E.G., Costigan, P.A., 2002. Static and dynamic biomechanics of foot orthoses in people with medial compartment knee osteoarthritis. Clin. Biomech. 17, 603-610.

Miyazaki, T., Wada, M., Kawahara, H., Sato, M., Baba, H., Shimada, S., 2002. Dynamic load at baseline can predict radiographic disease progression in medial compartment knee osteoarthritis. Ann. Rheum. Dis. 61 (7), 617-622. 
Mundermann, A., Dyrby, C.O., Andriacchi, T.P., 2005. Secondary gait changes in patients with medial compartment knee osteoarthritis: Increased load at the ankle, knee, and hip during walking. Arthritis Rheum. 52, 2835-2844.

Nakajima, K., Kakihana, W., Nakagawa, T., Mitomi, H., Hikita, A., Suzuki, R., Akai, M., Iwaya, T., Nakamura, K., Fukui, N., 2009. Addition of an arch support improves the biomechanical effect of a laterally wedged insole. Gait Posture 29, 208-213.

Reid, S.M., Lynn, S.K., Musselman, R.P., Costigan, P.A., 2007. Knee biomechanics of alternate stair ambulation patterns. Med. Sci. Sports Exerc. 39, 2005.

Rubin, R., Menz, H.B., 2005. Use of laterally wedged custom foot orthoses to reduce pain associated with medial knee osteoarthritis: a preliminary investigation. J. Am. Podiatr. Med. Assoc. 95, 347

Sasaki, T., Yasuda, K., 1987. Clinical evaluation of the treatment of osteoarthritic knees using a newly designed wedged insole. Clin. Orthop. Relat. Res. 221, 181.

Thorp, L.E., Sumner, D.R., Block, J.A., Moisio, K.C., Shott, S., Wimmer, M.A., 2006. Knee joint loading differs in individuals with mild compared with moderate medial knee osteoarthritis. Arthritis Rheum. 54, 3842-3849.
Tinetti, M.E., De Leon, C.F.M., Doucette, J.T., Baker, D.I., 1994. Fear of falling and fall-related efficacy in relationship to functioning among community-living elders. J. Gerontol. 49, M140.

Wallace, D.A., Pavol, M.J., Harter, R.A., 2007. Efficacy of lateral wedge orthotics during stair descent in patients with knee osteoarthritis. J. Biomech. 40, 677.

Walter, J.P., D'Lima, D.D., Colwell Jr., C.W., Fregly, B.J., 2010. Decreased knee adduction moment does not guarantee decreased medial contact force during gait. J. Orthop. Res. 28 (10), 1348-1354.

Whatling, G.M., Evans, S.L., Holt, C.A., 2009. Introducing a new staircase design to quantify healthy knee function during stair ascent and descent. Comput. Methods Biomech. Biomed. Eng. 13, 371-378.

Yasuda, K., Sasaki, T., 1987. The mechanics of treatment of the osteoarthritic knee with a wedged insole. Clin. Orthop. Relat. Res. 215, 162.

Zhao, D., Banks, S.A., Mitchell, K.H., D'Lima, D.D., Colwell Jr., C.W., Fregly, B.J., 2007. Correlation between the knee adduction torque and medial contact force for a variety of gait patterns. J. Orthop. Res. 25 (6), 789-797. 